\title{
A Preliminary Study on Dimension-Reduction Algorithm for Variational Methods in Three Dimensions
}

\author{
X. Chen \\ Numerical Analysis Research Center, GZ 510510, China
}

\begin{abstract}
Numerical weather prediction is an initial-value problem, for determination of the initial conditions, there are many methods and one of the most classical methods is variational methods in three dimensions, or 3D-Var. In this approach, with a defined cost function proportional to the square of the distance between the analysis and both the background and the observations, one can obtain the analysis. In the cost function, the background and the observations are reshaped to vectors; within this step, the order of the background error covariance matrix and the observational error covariance matrix becomes huge, which is not convenient to one to obtain the analysis. In this paper, according to the matrix analysis approach, we put forward some possible improvements to the dimension-reduction algorithm of 3D-Var, so that provide some references for data assimilation.
\end{abstract}

Key words: 3D-Var; matrix; dimension-reduction algorithm

\section{Introduction}

On the basis of Bayes' theorem, Lorenc (1986) gave an analysis method for estimating the initial-value, which is now known as 3D-Var and widely used all over the world (Chouinard, 2003; Brousseau et al., 2015; Liu et al., 2016; Zaripov et al., 2016; Wang et al., 2017). In his paper, Lorenc laid the mathematical foundation of 3D-Var. In this famous approach, the cost function is defined as:

$$
J(\mathbf{x})=\left(\mathbf{x}-\mathbf{x}_{b}\right)^{\mathrm{T}} \mathbf{B}^{-1}\left(\mathbf{x}-\mathbf{x}_{b}\right)+(\mathbf{y}-\mathbf{H}[\mathbf{x}])^{\mathrm{T}} \mathbf{R}^{-1}(\mathbf{y}-\mathbf{H}[\mathbf{x}]),
$$

where $\mathbf{B}$ is the background error covariance matrix, $\mathbf{R}$ is the observational error covariance matrix, $\mathbf{x}$ is the vector of the analysis, $\mathbf{x}_{b}$ is the vector of the background or the first guess, $\mathbf{y}$ is the vector of the observation and $\mathbf{H}[]$ is the observation operator. The cost function is minimized directly to

\footnotetext{
*Corresponding author: Chen Xuan (E-mail: chzffx@qq.com or chenxuan@mail.iap.ac.cn)
} 
obtain the analysis of the atmospheric or oceanic state at a given time. As the dimension of $\mathbf{B}$ and $\mathbf{R}$ is too large, there are a lot of papers (Bannister, 2008a; Bannister, 2008b; Cohn et al., 1996; Farrell et al., 2010) for solving equation (1); some of these methods are applied by many centers, such as NMC (National Meteorological Center) method (Parrish et al., 1992), which is used for estimating the forecast error covariance.

The minimum of cost function in equation (1) is attained for $\mathbf{x}=\mathbf{x}_{a}$ (the vector of analysis), the analysis is given by the solution of:

$$
\nabla J(\mathbf{x})=2 \mathbf{B}^{-1}\left(\mathbf{x}-\mathbf{x}_{b}\right)+2 \mathbf{H}^{\mathrm{T}} \mathbf{R}^{-1} \mathbf{H}\left(\mathbf{x}-\mathbf{x}_{b}\right)-2 \mathbf{H}^{\mathrm{T}} \mathbf{R}^{-1}\left(\mathbf{y}-\mathbf{H}\left[\mathbf{x}_{b}\right]\right) \equiv \mathbf{0} .
$$

By solving this equation, we can obtain $\mathbf{x}_{a}$ :

$$
\mathbf{x}_{a}=\mathbf{x}_{b}+\Delta
$$

where $2 \mathbf{B}^{-1} \boldsymbol{\Delta}+2 \mathbf{H}^{\mathrm{T}} \mathbf{R}^{-1} \mathbf{H} \boldsymbol{\Delta}-2 \mathbf{H}^{\mathrm{T}} \mathbf{R}^{-1}\left(\mathbf{y}-\mathbf{H}\left[\mathbf{x}_{b}\right]\right) \equiv \mathbf{0}$.

In general, all of these methods mentioned in the papers cited above or not are without dimension-reduction of $\mathbf{B}$ and $\mathbf{R}$, so the dimension of the matrixes is also too large. For example, in 3D-Var, a variance field of dimension $(m \times n)$ is corresponded to the matrix $\mathbf{B}$ of dimension $(m n \times m n)$; for the global model or high resolution model, this dimension is a "terrible" magnitude.

\section{Dimension-reduction algorithm}

By analyzing the structure of the cost function, equation (1), we can find that, the cost function $J(\mathbf{x})$ can be rewritten as $J(\mathbf{X})$ :

$$
J(\mathbf{X})=J_{1}(\mathbf{X})+J_{2}(\mathbf{X})+J_{3}(\mathbf{X})+J_{4}(\mathbf{x}),
$$

where, $J_{i}(\mathbf{X})(\mathrm{i}=1,2,3,4)$ is the trace of the function of the background or the observation, for example:

$$
J_{1}(\mathbf{x})=\operatorname{Trace}\left[\left(\mathbf{X}-\mathbf{X}_{b}\right)^{\mathbf{T}} \mathbf{B}_{1}^{-1}\left(\mathbf{X}-\mathbf{X}_{b}\right)\right]
$$

where the operator "Trace[]" is used for calculating the trace of the matrix; and $J_{2}(\mathbf{X})$ is similar to $J_{1}(\mathbf{X}), J_{3}(\mathbf{X})$ and $J_{4}(\mathbf{X})$ are similar and used for calculating the trace for the part of observations. On the basis of this thought, we can transform the processes for solving the equation (1) to solving the equation (2), in which the background error covariance matrixes are of a dimension similar to the background. So, there are four convariance matrixes: two background error covariance matrixes 
( $\mathbf{B}_{1}$ and $\mathbf{B}_{2}$ ) and two observational error covariance matrixes $\left(\mathbf{R}_{1}\right.$ and $\mathbf{R}_{2}$ ). All matrixes are square matrixes, for example, for a variance of a dimension $(m \times n)$, the matrix $\mathbf{B}_{1}$ is of a dimension $(m \times m)$, the matrix $\mathbf{B}_{2}$ is of a dimension $(n \times n)$, and same to the matrixes $\mathbf{R}_{1}$ and $\mathbf{R}_{2}$. For the observation operator $\mathbf{H}$, on the basis of matrix theory and more generalized linear regression model (Chen et al., 2017), we can set that, $\mathbf{H}[\mathbf{x}] \sim \mathbf{H}[\mathbf{X}]$ (which means $\mathbf{H}[\mathbf{X}]$ is equivalent to $\mathbf{H}[\mathbf{x}]$ ), and for a linear observation operator, $\mathbf{H}[\mathbf{X}]$ can be written as $\mathbf{H}[\mathbf{X}] \equiv \mathbf{A X D}$.

In Hilbert space, we can make a reasonable assumption that there must be observation operators $\mathbf{A}$ and $\mathbf{D}$, background error covariance matrixes $\mathbf{B}_{1}$ and $\mathbf{B}_{2}$, observational and representative error covariance matrixes $\mathbf{R}_{1}$ and $\mathbf{R}_{2}$, which satisfy equation (2), with the same analysis, which is the solution of equation (1).

In this paper, we will discuss a simple situation, in which the observation is adopted at each point in the calculating grids. So, for a variance observation of a dimension $(m \times n)$, the matrix $\mathbf{R}_{1}$ is of a dimension $(m \times m)$, and the matrix $\mathbf{R}_{2}$ is of a dimension $(n \times n)$.

\subsection{Discussion in the simple situation}

The key point for obtaining the analysis from equation (2) is to get the derivative of $J_{i}(\mathbf{X})$ $(\mathrm{i}=1,2,3,4)$.

Differentiating equations (3) with respect to $\mathbf{X}$ gives:

$$
\frac{\partial J_{1}(\mathbf{X})}{\partial \mathbf{X}}=\mathbf{B}_{1}^{-1} \mathbf{X}+\mathbf{B}_{1}^{-1 \mathbf{T}} \mathbf{X}-\mathbf{B}_{1}^{-1} \mathbf{X}_{b}-\mathbf{B}_{1}^{-1 \mathbf{T}} \mathbf{X}_{b}
$$

Similar to other terms, and substituting these equations following equation:

$$
\frac{\partial J(\mathbf{x})}{\partial \mathbf{X}}=\frac{\partial}{\partial \mathbf{X}}\left[J_{1}(\mathbf{x})+J_{2}(\mathbf{x})+J_{3}(\mathbf{x})+J_{4}(\mathbf{x})\right]
$$

we can get:

$$
\frac{\partial J(\mathbf{X})}{\partial \mathbf{X}}=\mathbf{B}_{1 S} \mathbf{X}+\mathbf{A}^{\mathrm{T}} \mathbf{R}_{1 \mathrm{~S}} \mathbf{A} \mathbf{X} \mathbf{U}^{\mathrm{T}} \boldsymbol{\Lambda}_{\mathbf{D}} \mathbf{U}+\mathbf{X} \mathbf{B}_{2 \mathrm{~S}}+\mathbf{V}^{\mathrm{T}} \boldsymbol{\Lambda}_{\mathbf{A}} \mathbf{V X D R}_{2 \mathrm{~S}} \mathbf{D}^{\mathrm{T}}-\mathbf{F}_{1} \mathbf{U}-\mathbf{V}^{\mathrm{T}} \mathbf{F}_{2}
$$

where $\mathbf{G}=\mathbf{Y}-\mathbf{A} \mathbf{X}_{b} \mathbf{D}, \mathbf{D D}^{\mathbf{T}}=\mathbf{U}^{\mathbf{T}} \boldsymbol{\Lambda}_{\mathbf{D}} \mathbf{U}, \mathbf{A}^{\mathbf{T}} \mathbf{A}=\mathbf{V}^{\mathbf{T}} \boldsymbol{\Lambda}_{\mathbf{A}} \mathbf{V}, \mathbf{U}^{\mathbf{T}} \mathbf{U}=\mathbf{V}^{\mathbf{T}} \mathbf{V}=\mathbf{U U}^{\mathbf{T}}=\mathbf{V}^{\mathbf{T}}=\mathbf{I}$ (I is unit matrix), and the other terms are:

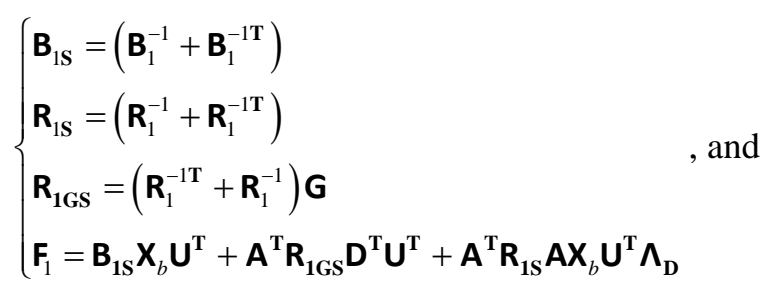




$$
\left\{\begin{array}{l}
\mathbf{B}_{2 \mathbf{S}}=\left(\mathbf{B}_{2}^{-1}+\mathbf{B}_{2}^{-1 \mathbf{T}}\right) \\
\mathbf{R}_{2 \mathbf{S}}=\left(\mathbf{R}_{2}^{-1}+\mathbf{R}_{2}^{-1 \mathbf{T}}\right) \\
\mathbf{R}_{2 \mathbf{G S}}=\mathbf{G}\left(\mathbf{R}_{2}^{-1}+\mathbf{R}_{2}^{-1 \mathbf{T}}\right) \\
\mathbf{F}_{2}=\mathbf{V} \mathbf{X X}_{b} \mathbf{B}_{2 \mathbf{S}}+\mathbf{V} \mathbf{A}^{\mathbf{T}} \mathbf{R}_{2 \mathbf{G S}} \mathbf{D}^{\mathbf{T}}+\boldsymbol{\Lambda}_{\mathbf{A}} \mathbf{V} \mathbf{X}_{b} \mathbf{D} \mathbf{R}_{2 \mathbf{S}} \mathbf{D}^{\mathbf{T}}
\end{array} .\right.
$$

The analysis can be obtained by equation (4):

$$
B_{1 S} \Delta+\Delta B_{2 S}+A^{T} R_{1 S} A \Delta D D^{T}+A^{T} A \Delta D R_{2 S} D^{T}=A^{T}\left(R_{1 G S}+R_{2 G S}\right) D^{T},
$$

where $\boldsymbol{\Delta}=\mathbf{X}-\mathbf{X}_{b}$. Equation (4) is similar to Sylvester equation $(\mathbf{A X}+\mathbf{X B}+\mathbf{C}=\mathbf{0})$, and we can adopt the iterative approach for solving this equation, then we can get the analysis $\mathbf{X}_{a}, \mathbf{X}_{a}=\mathbf{X}_{b}+\Delta$.

Sylvester equation is a matrix equation of the form $(\mathbf{A X}+\mathbf{X B}+\mathbf{C}=\mathbf{0})$; with the given matrixes $\mathbf{A}$, $\mathbf{B}$, and $\mathbf{C}$, the problem is to find the possible matrixes $\mathbf{X}$ that obey this equation. This equation has a wide range of applications in many fields, such as cybernetics. In the simple case, where the observation operators are unit matrix, equation (4) can be reduced to Sylvester equation. In section 2.2, we will show this situation, and a numerical experiment based on Sylvester equation will be given in section 2.3 .

\subsection{Equation (4) in the simple situation $(A=D=1)$}

In the simple situation, we can take $\mathbf{Y}-\mathbf{X}_{b}$ as $\mathbf{G}$, as $\mathbf{A}=\mathbf{D}=\mathbf{I}$. So the equation (4) can be written as:

$$
\left(\mathbf{B}_{\mathbf{R} 1}+\mathbf{B}_{\mathbf{R} 1}^{\mathbf{T}}\right) \boldsymbol{\Delta}+\boldsymbol{\Delta}\left(\mathbf{B}_{\mathbf{R} 2}+\mathbf{B}_{\mathbf{R} 2}^{\mathbf{T}}\right)-\left(\mathbf{R}_{1}^{-1 \mathbf{T}}+\mathbf{R}_{1}^{-1}\right) \mathbf{G}-\mathbf{G}\left(\mathbf{R}_{2}^{-1 \mathbf{T}}+\mathbf{R}_{2}^{-1}\right)=\mathbf{0},
$$

where, $\boldsymbol{\Delta}=\mathbf{X}-\mathbf{X}_{b}, \quad \mathbf{B}_{\mathbf{R} 1}=\mathbf{B}_{1}^{-1}+\mathbf{R}_{1}^{-1}$ and $\mathbf{B}_{\mathbf{R} 2}=\mathbf{B}_{2}^{-1}+\mathbf{R}_{2}^{-1}$. Equation (5) is Sylvester equation with given matrixes $\mathbf{B}_{\mathbf{R} 1}+\mathbf{B}_{\mathbf{R} 1}^{\mathbf{T}}, \mathbf{B}_{\mathbf{R} 2}+\mathbf{B}_{\mathbf{R} 2}^{\mathbf{T}}$, and $-\left(\mathbf{R}_{1}^{-1 \mathbf{T}}+\mathbf{R}_{1}^{-1}\right) \mathbf{G}-\mathbf{G}\left(\mathbf{R}_{2}^{-1 \mathbf{T}}+\mathbf{R}_{2}^{-1}\right)$ as matrixes $\mathbf{A}$, $\mathbf{B}$, and $\mathbf{C}$ in Sylvester equation $(\mathbf{A} \mathbf{X}+\mathbf{X} \mathbf{B}+\mathbf{C}=\mathbf{0}$, here the matrixes $\mathbf{A}, \mathbf{B}$, and $\mathbf{C}$ are not matrixes in the observation operator). The problem of obtaining the analysis satisfies the cost function is transformed to solve the equation (5).

With MATLAB, we can adopt the function "lyap" for solving Sylvester equation, then, we can get the analysis, $\mathbf{X}_{a}=\mathbf{X}_{b}+\boldsymbol{\Delta}$. There are a lot of methods for solving this equation, and here we will not go into details.

In the simple situation and equation (1), we can get the analysis with vector form: 


$$
\left(\mathbf{B}^{-1}+\mathbf{R}^{-1}\right) \Delta-\mathbf{R}^{-1} \mathbf{g}=\mathbf{0},
$$

where $\boldsymbol{\Delta}=\mathbf{x}-\mathbf{x}_{b}$, and $\mathbf{g}=\mathbf{y}-\mathbf{x}_{b}$. Using the Kronecker product notation and the vectorization operator "vec", we can rewrite Sylvester equation in the form:

$$
\left[\mathbf{I} \otimes\left(\mathbf{B}_{\mathbf{R} 1}+\mathbf{B}_{\mathbf{R} 1}^{\mathbf{T}}\right)+\left(\mathbf{B}_{\mathbf{R} 2}+\mathbf{B}_{\mathbf{R} 2}^{\mathbf{T}}\right)^{\mathbf{T}} \otimes \mathbf{I}\right] \operatorname{vec}(\boldsymbol{\Delta})=\operatorname{vec}\left[\left(\mathbf{R}_{1}^{-1 \mathbf{T}}+\mathbf{R}_{1}^{-1}\right) \mathbf{G}+\mathbf{G}\left(\mathbf{R}_{2}^{-1 \mathbf{T}}+\mathbf{R}_{2}^{-1}\right)\right],
$$

in this form, the equation can be seen as a linear system of dimension $(m n \times m n)$, where the variance $\mathbf{X}$ is of a dimension $(m \times n)$. We can find that both equations (6) and (7) are similar.

For a data assimilation system with $3 \mathrm{D}-\mathrm{Var}$ or $4 \mathrm{D}$-Var, it is important to estimate the background error covariance matrix(es). By estimating the background error covariance matrix(es), we can use these matrix(es) to solving equation (5) or (6).

When running a numerical weather prediction system for a small forecast region, or with low resolution, equation (6) can be applied. For example, with a dimension of (50×50), in this case, the matrixes (B and $\mathbf{R}$ ) are of dimension $(2500 \times 2500)$, and most personal computers can work well in this situation. When we set the dimension as $(100 \times 100)$, the matrixes $(\mathbf{B}$ and $\mathbf{R})$ are of dimension $(10000 \times 10000)$, for most personal computers, it is hard to allocate enough memory for calculating.

For the high resolution global numerical models, the resolution can reach $0.125^{\circ}$. The dimension of the computing grid in these models can be larger than $(2880 \times 1441)$; in this situation, the matrixes (B and $\mathbf{R})$ in equation (6) are with a dimension of $(4150080 \times 4150080)$. So, for supercomputer, it is also hard to allocate memory for these matrixes, and there is no direct method to solve equation (6) in this case. But, the matrixes $\left(\mathbf{B}_{1}, \mathbf{B}_{2}, \mathbf{R}_{1}, \mathbf{R}_{2}, \mathbf{A}\right.$ and $\left.\mathbf{D}\right)$ are with a dimension of (2880×1441), which means that if the model can be run, the 3D-Var approach can also be adopted with equation (5) through a direct method.

\subsection{Numerical experiments in the simple situation $(A=D=I)$}

In this section, we will present numerical experiments in the simple situation $(\mathbf{A}=\mathbf{D}=\mathbf{I})$. On the basis of equation (5) (also known as Sylvester equation), the result are shown in Fig. 1 and table 1. As mentioned above, this situation means that the observation is adopted at each point in the calculating grids. The background error statistics on 3D-Var is a very important issues (Rakesh \& Goswami, 2011; Sahu et al., 2014), here as the covariance matrixes is un-similar to the matrixes 
in equation(1), we will apply the way to construct the error covariance matrixes $\left(\mathbf{B}_{i}, \mathbf{R}_{i}, i=1,2\right)$ which can be found by this work (Chen et al. 2017).

In Fig. 1, the ideal field is produced by "peaks" in MATLAB with a dimension of $(100 \times 100)$ (the result is shown in table 1 , where MAE represents the maximum absolute error, RMSE represents the root mean square error, MAE1 represents the mean absolute error, and ME represents the mean error; the comparison of equations (5) and (6) are shown in table 2 with a dimension of $(40 \times 40))$, and shown in a in Fig. 1; the background is shown in b, which is produced by superimposed random error of normal distribution on the ideal fields (same to experiments in table 2); the observation is shown in c, which is also produced by superimposed random error of normal distribution on the ideal fields (same to experiments in table 2); the analysis is shown in d, which obey equation (5).
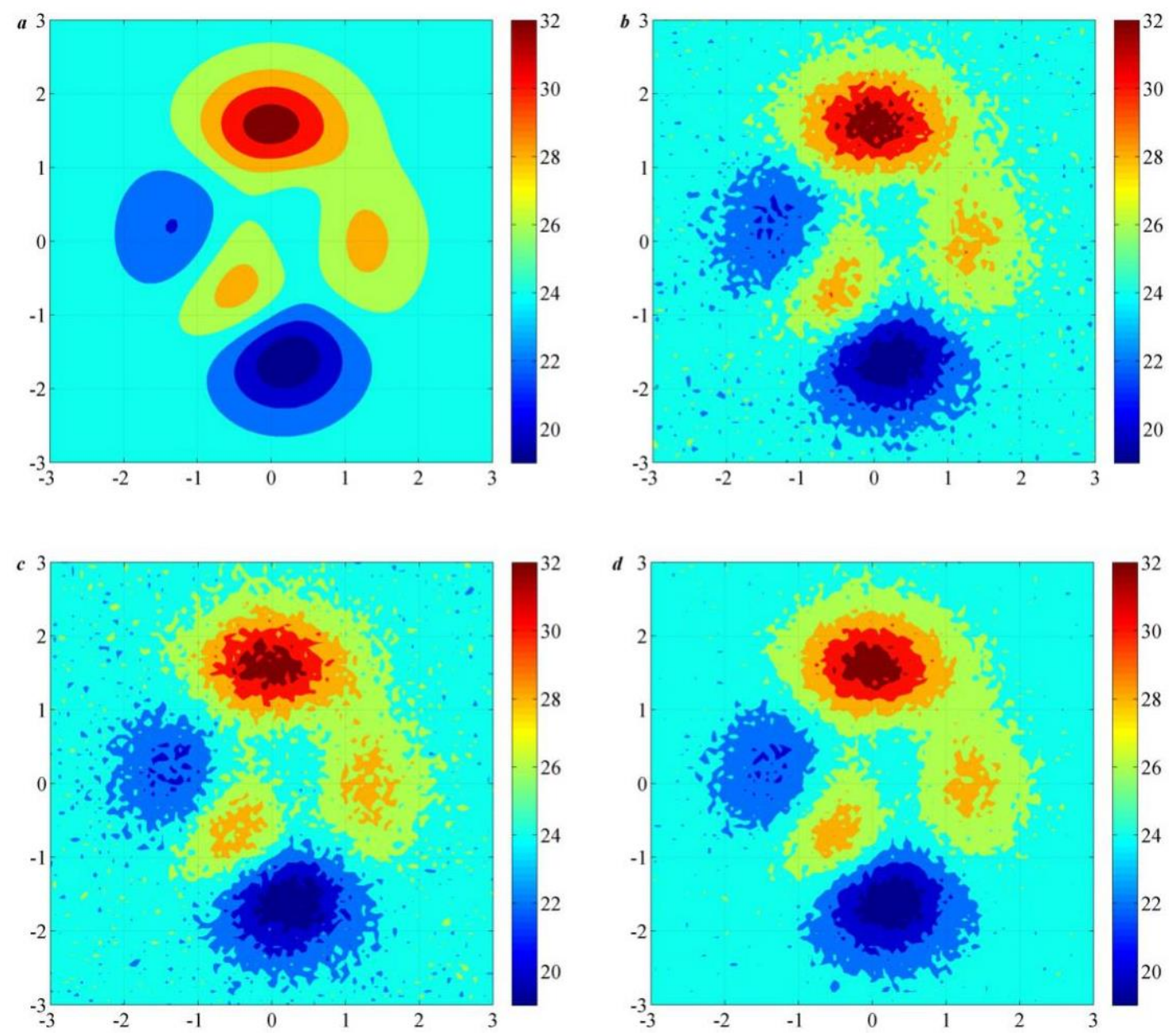

Fig. 1 The result of numerical experiment with a dimension of $(100 \times 100)$ 
Table 1 The result of numerical experiment with a dimension of $(100 \times 100)$

\begin{tabular}{ccccc}
\hline & MAE & RMSE & MAE1 & ME \\
\hline background field & 2.7890 & 0.6756 & 0.5400 & -0.0121 \\
observation field & 2.9519 & 0.7196 & 0.5756 & 0.0061 \\
analysis field & 1.8670 & 0.4927 & 0.3925 & -0.0036 \\
\hline
\end{tabular}

Table 2 The result of numerical experiment with a dimension of $(40 \times 40)$

\begin{tabular}{ccccc}
\hline & MAE & RMSE & MAE1 & ME \\
\hline background field & 2.9268 & 0.8902 & 0.7135 & -0.0029 \\
observation field & 2.9832 & 0.9196 & 0.7422 & -0.0173 \\
analysis field (eqution (5)) & 2.0961 & 0.6437 & 0.5174 & -0.0173 \\
analysis field (eqution (6)) & 2.5018 & 0.7282 & 0.5851 & -0.0169 \\
\hline
\end{tabular}

The result (in table 1 and 2) shows that, for the similar type of error, equation (5) is applicable. In table 1, comparing the analysis and the observation, the RMSE dropped about 0.23, the MAE1 dropped about 0.18; comparing the analysis and the background, the RMSE dropped about 0.19 , the MAE1 dropped about 0.14 . The analysis' mean error is near to zero, and there is no significant abnormal value distribution in the analysis with comparing to the ideal field. In table 2 , the comparison results show that the analysis obtained by equation (5) is better than the one obtained by equation (6).

The dimension of all matrixes in equation $(5)$ is only $(100 \times 100)$, which can be run with personal computers. As discussion above, the dimension of background error covariance matrix is with a dimension of $(10000 \times 10000)$ in equation $(6)$, which is hard for a personal computer to allocate memory for computing.

\section{Summary}

As discussed above, equation (2) is very practical, and this equation is more applicable for grid observation and can be also applied in data assimilation with conventional observation. With the calculation results in section 2.3, the assimilation effect is achieved in the numerical experiments based on equation (2), which significantly reduces the computational magnitude. But, there are also some problems (Bannister, 2008a; Bannister, 2008b): how to construct the background error covariance matrixes $\mathbf{B}_{1}$ and $\mathbf{B}_{2}$ and observational error covariance matrixes $\mathbf{R}_{1}$ and $\mathbf{R}_{2}$ is also an important problem. 


\section{References}

A. C. Lorenc. 1986. Analysis methods for numerical weather prediction. Quarterly Journal of the Royal Meteorological Society, 112: 1177-1194.

B. F. Farrell, P. J. Ioannu. 2010. State estimation using a reduced order Kalman filter. Journal of the Atmospheric Sciences, 58(23): 3666-3680.

C. Chouinard. 2003. Use of moisture sensitive satellite radiances in the Canadian Meteorological Centre Unified 3D-Var system. Proceedings of SPIE-The International Society for Optical Engineering 4895, doi: 10.1117/12/466839.

Chen Xuan, Zheng Chongwei, Zhang Weitao, Li Xin, Jin Peng. 2017. More generalized linear regression model and its meteorological application. Journal of PLA University of Science and Technology Natural Science Edition, 18(2): 144-149.

D. F. Parrish, J. D. Derber. 1992. The National Meteorological Center spectral statistical interpolation analysis system. Monthly Weather Review, 120: 1747-1763.

D. K. Sahu, S. K. Dash, S. C. Bhan. 2014. Impact of surface observations on simulation of rainfall over NCR Delhi using regional background error statistics in WRF-3DVAR model. Meteorology \& Atmospheric Physics, 125 (1-2): 17-42.

P. Brousseau, L. Berre, F. Bouttier, G. Desroziers. 2015. Background-error covariances for a convective-scale data-assimilation system: AROME-France 3D-Var. 137 (655): 409-422.

R. B. Zaripov, Y. V. Martynova, V. N. Krupchatnikov, A. P. Petrov. 2016. Atmosphere data assimilation system for the Siberian region with the WRF-ARW model and three-dimensional variational analysis WRF 3D-Var. Russian Meteorology \& Hydrology, 41 (11-12): 808-815.

R. N. Bannister. 2008a. A review of forecast error covariance statistics in atmospheric variational data assimilation. I: Characteristics and measurements of forecast error covariances. Quarterly Journal of the Royal Meteorological Society, 134: 1951-1970.

R. N. Bannister. 2008b. A review of forecast error covariance statistics in atmospheric variational data assimilation. II: Modelling the forecast error covariance statistics. Quarterly Journal of the Royal Meteorological Society, 134: 1971-1996.

S. E. Cohn, R. Todling. 1996. Appropriate data assimilation schemes for stable and unstable dynamics. Journal of the Meteorological Society of Japan, 74(1): 63-75.

V. Rakesh, P. Goswami. 2011. Impact of background error statistics on 3D-Var assimilation: 
case study over the Indian region. Meteorology \& Atmospheric Physics, 112 (1-2): 63-79.

Liu Yan, Xue Jishan, Zhang Lin, Lu Huijuan. 2016. Verification and diagnostics for Data Assimilation System of Global GRAPES. Journal of Applied Meteorological Science, 27 (1): $1-15$.

Wang Jincheng, Lu Huijuan, Han Wei, Liu Yan, Wang Ruichun, Zhang Hua, Huang Jing, Liu Yongzhu, Hao Min, Li Juan, Tian Weihong. 2017. Improvements and performances of the operational GRAPES GFS 3DVar system. Journal of Applied Meteorological Science, 28 (1): $11-24$. 